Article

\title{
Existence Results for the Solution of the Hybrid Caputo-Hadamard Fractional Differential Problems Using Dhage's Approach
}

\author{
Muhammad Yaseen ${ }^{1}$ (D), Sadia Mumtaz ${ }^{1}$, Reny George ${ }^{2, *(D)}$ and Azhar Hussain ${ }^{1}$ (D) \\ 1 Department of Mathematics, University of Sargodha, Sargodha 40100, Pakistan; \\ yaseen.yaqoob@uos.edu.pk (M.Y.); mumtazsadia6@gmail.com (S.M.); azhar.hussain@uos.edu.pk (A.H.) \\ 2 Department of Mathematics, College of Science and Humanities in Al-Kharj, Prince Sattam bin Abdulaziz \\ University, Al-Kharj 11942, Saudi Arabia \\ * Correspondence: renygeorge02@yahoo.com
}

Citation: Yaseen, M.; Mumtaz, S.; George, R.; Hussain, A. Existence Results for the Solution of the Hybrid Caputo-Hadamard Fractional Differential Problems Using Dhage's Approach. Fractal Fract. 2022, 6, 17. https://doi.org/10.3390/ fractalfract6010017

Academic Editor: Mark Edelman

Received: 6 November 2021

Accepted: 24 December 2021

Published: 30 December 2021

Publisher's Note: MDPI stays neutral with regard to jurisdictional claims in published maps and institutional affiliations.

Copyright: (C) 2021 by the authors. Licensee MDPI, Basel, Switzerland. This article is an open access article distributed under the terms and conditions of the Creative Commons Attribution (CC BY) license (https:// creativecommons.org/licenses/by/ $4.0 /)$.
Abstract: In this work, we explore the existence results for the hybrid Caputo-Hadamard fractional boundary value problem (CH-FBVP). The inclusion version of the proposed BVP with a three-point hybrid Caputo-Hadamard terminal conditions is also considered and the related existence results are provided. To achieve these goals, we utilize the well-known fixed point theorems attributed to Dhage for both BVPs. Moreover, we present two numerical examples to validate our analytical findings.

Keywords: Caputo-Hadamard fractional derivative; thermostat modeling; Caputo-Hadamard fractional integral; hybrid Caputo-Hadamard fractional differential equation and inclusion

\section{Introduction}

Fractional differential equations are utilized for mathematical modeling of real life problems. Scientists working in various fields of science are encouraged to improve the explanation of their findings by including more accurate knowledge into their problems. In this regard, they are employing a variety of mathematical methods in their models in which fractional order derivatives are very beneficial. Differential equations of a fractional order provide more accurate information than standard differential equations in mathematical modeling of many scientific situations. In these days, differential equations of a fractional order have been constantly utilized in chemistry, biophysics, control theory, mechanics, image processing, polymer rheology, aerodynamics, etc. [1,2].

In recent years, many researchers have been attracted by fractional hybrid differential equations and inclusions with terminal conditions [3-6]. Moreover, in various fields, there are several efforts on the Caputo-Hadamard derivative of fractional order and its implementations [7-15].

In 2010, a new class of differential models named hybrid differential equations (HDEs) was formulated by Dhage and Lakshmikantham [16]. Moreover, they investigated properties of this type of a differential equation's solution. Zhao et al. [17] generalized Dhage's effort and investigated the related HDEs of fractional order. In 2012, a fractional hybrid problem with two-point terminal conditions was presented by Sun et al. [18]

$$
\left\{\begin{array}{l}
\mathfrak{D}_{0}^{w}\left[\frac{s(\ell)}{g(\ell, s(\ell))}\right]+w(\ell, s(\ell))=0, \quad \ell \in \mathcal{E}=[0,1], w \in(1,2], \\
s(0)=s(1)=0 .
\end{array}\right.
$$

They obtained some existence results by using a fixed point theorem presented by Dhage in Banach algebra with Lipschitz and mixed Caratheodory conditions. In 2015, the 
existence criteria for the solutions of the hybrid Caputo problem with terminal conditions was studied by Hilal and Kajouni [19]

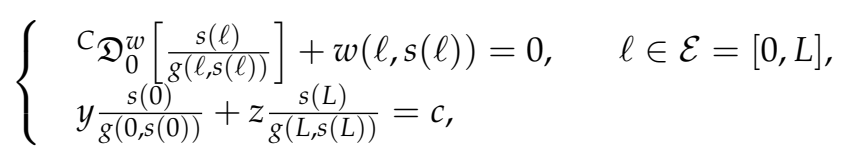

where, $w \in(0,1), y, z, c \in \Re$ with $y+z \neq 0$ and $g: \mathcal{E} \times \Re \longrightarrow \Re \backslash 0$ and $w: \mathcal{E} \times \Re \longrightarrow \Re$ are continuous functions. Baleanu et al. [20] studied the existence criteria and significance of the solution for a new kind of hybrid inclusion problem of fractional order,

$$
c_{\mathfrak{D}_{0}^{w}}^{w}\left(\frac{s(\ell)}{\left.g(\ell, s(\ell)), \Im_{0}^{p_{1}} s(\ell), \ldots, \Im_{0}^{p_{t}} s(\ell)\right)}\right) \in \mathcal{Q}\left(\ell, s(\ell), \Im_{0}^{q_{1}} s(\ell), \ldots, \Im_{0}^{q_{u}} s(\ell)\right), \quad \ell \in[0,1]
$$

equipped with boundary conditions $s(0)=s_{0}^{*}$ and $s(1)=s_{1}^{*}$, where, $w \in(1,2], \Im_{0}^{\beta}$ and ${ }^{C} \mathfrak{D}_{0}^{w}$ symbolize the Riemann-Liouville fractional integral operator of order $\beta \in\left\{p_{k}, q_{j}\right\} \subset(0, \infty)$ for $k=1, \ldots, t$ and $j=1,2, \ldots, u$ and a fractional Caputo derivative of order $w$, respectively. In 2006, a thermostat model enclosed at $\ell=0$ with a restrainer at $\ell=1$ was studied by Infante and Webb [21],

$$
\left\{\begin{array}{l}
s^{\prime \prime}(\ell)+v(\ell, s(\ell))=0, \quad 0 \leq \ell \leq 1 \\
s^{\prime}(0)=0, \quad \tau s^{\prime}(1)+s(\zeta)=0
\end{array}\right.
$$

where, $\zeta \in[0,1]$ is a real constant and $\tau>0$ is a positive number. The thermostat includes or excludes heat based on the temperature exposed by the sensor at $\ell=\zeta$ by using this second order approach. They applied a fixed point criteria on Hammerstein integral equations to obtain the existence results for the above BVP. A fractional order problem was presented by Nieto and Pimentel [22],

$$
\left\{\begin{array}{l}
C_{\mathfrak{D}}^{w} s(\ell)+v(\ell, s(\ell))=0, \quad \ell \in[0,1] \\
s^{\prime}(0)=0, \quad \tau^{C} \mathfrak{D}_{0}^{w-1} s(1)+s(\zeta)=0
\end{array}\right.
$$

where, $\zeta \in[0,1], \tau>0$ is any positive real number and ${ }^{C} \mathfrak{D}_{0}^{w}$ represents the fractional Caputo derivative of order $w \in(1,2]$. It is noticeable that a thermostat is a main component which plays an important role in physical systems to maintain its temperature near a required set-point, which motivated many researchers to study the various models of thermostat systems. In 2020, Baleanu et al. [4] constructed the following Caputo fractional hybrid problem for thermostat model,

$$
C_{\mathfrak{D}_{0}^{w}}\left(\frac{s(\ell)}{g(\ell, s(\ell))}\right)+v(\ell, s(\ell))=0, \quad \ell \in[0,1],
$$

supplemented with the hybrid terminal conditions,

$$
\left\{\begin{array}{l}
\left.\mathfrak{D}\left(\frac{s(\ell)}{g(\ell, s(\ell))}\right)\right|_{\ell=0}=0, \\
\left.\tau^{C} \mathfrak{D}_{0}^{w-1}\left(\frac{s(\ell)}{g(\ell, s(\ell))}\right)\right|_{\ell=1}+\left.\left(\frac{s(\ell)}{g(\ell, s(\ell))}\right)\right|_{\ell=\zeta}=0,
\end{array}\right.
$$

where, $w \in(1,2], w-1 \in(0,1], \mathfrak{D}=\frac{d}{d \ell}, \tau$ is any positive real number, $\zeta \in[0,1]$ and ${ }^{C} \mathfrak{D}_{0}^{\beta}$ is the fractional Caputo derivative of order $\beta \in\{w, w-1\}$. Furthermore, $v: \mathcal{E} \times \Re \rightarrow \Re$ and $g: \mathcal{E} \times \Re \rightarrow \Re \backslash\{0\}$ are continuous functions. Moreover, they studied the related hybrid Caputo inclusion model of a fractional order for a thermostat system, as given below:

$$
C_{\mathfrak{D}_{0}^{w}}\left(\frac{s(\ell)}{g(\ell, s(\ell))}\right) \in \mathfrak{\omega}(\ell, s(\ell)), \quad \ell \in \mathcal{E}=[0,1],
$$


supplemented with three-point hybrid Caputo terminal conditions,

$$
\left\{\begin{array}{l}
\left.\mathfrak{D}\left(\frac{s(\ell)}{g(\ell, s(\ell))}\right)\right|_{\ell=0}=0, \\
\left.\tau^{\mathcal{C}} \mathfrak{D}_{0}^{w-1}\left(\frac{s(\ell)}{g(\ell, s(\ell))}\right)\right|_{\ell=1}+\left.\left(\frac{s(\ell)}{g(\ell, s(\ell))}\right)\right|_{\ell=\zeta}=0,
\end{array}\right.
$$

where, $\omega: \mathcal{E} \times \Re \rightarrow \mathcal{I}(\Re)$ is a multi-valued map. Motivated by the previous studies, we construct the following hybrid CH-FBVP for thermostat model

$$
{ }^{C H} \mathfrak{D}_{1^{+}}^{w}\left(\frac{s(\ell)}{g(\ell, s((\ell))}\right)+\eta(\ell, s(\ell))=0, \quad \ell \in[1, e],
$$

supplemented with the three-point hybrid terminal conditions,

$$
\left\{\begin{array}{l}
\left.\mathfrak{D}\left(\frac{s(\ell)}{g(\ell, s(\ell))}\right)\right|_{\ell=1}=0, \\
\left.\tau^{C H} \mathfrak{D}_{1^{+}}^{w-1}\left(\frac{s(\ell)}{g(\ell, s(\ell))}\right)\right|_{\ell=e}+\left.\left(\frac{s(\ell)}{g(\ell, s(\ell))}\right)\right|_{\ell=\zeta}=0,
\end{array}\right.
$$

where, $w \in(1,2], w-1 \in(0,1], \mathfrak{D}=\frac{d}{d \ell}, \tau \in \Re^{+}, \zeta \in[1, e]$ and ${ }^{C H} \mathfrak{D}_{1^{+}}^{\alpha}$ is the fractional Caputo-Hadamard derivative of order $\alpha \in\{w, w-1\}$. Moreover, $\eta: \mathcal{E} \times \Re \rightarrow \Re$ and $g: \mathcal{E} \times \Re \rightarrow \Re \backslash\{0\}$ are continuous functions. Moreover, we study the related hybrid Caputo-Hadamard fractional inclusion boundary value problem (CH-FIBVP) for thermostat system as given below:

$$
{ }^{C} H_{\mathfrak{D}_{1+}}^{w}\left(\frac{s(\ell)}{g(\ell, s(\ell))}\right) \in \mathfrak{\omega}(\ell, s(\ell)), \quad \ell \in \mathcal{E}=[1, e]
$$

supplemented with three-point hybrid Caputo-Hadamard boundary conditions

$$
\left\{\begin{array}{l}
\left.\mathfrak{D}\left(\frac{s(\ell)}{g(\ell, s(\ell))}\right)\right|_{\ell=1}=0, \\
\left.\tau^{C H} \mathfrak{D}_{1^{+}}^{w-1}\left(\frac{s(\ell)}{g(\ell, s(\ell))}\right)\right|_{\ell=e}+\left.\left(\frac{s(\ell)}{g(\ell, s(\ell))}\right)\right|_{\ell=\zeta}=0,
\end{array}\right.
$$

where, $\mathscr{\omega}:[1, e] \times \Re \rightarrow \mathcal{I}(\Re)$ is a multi-valued map.

The main motivation behind this work is that there are no research manuscripts based on the authors' knowledge on the problems involving Caputo-Hadamard hybrid fractional boundary conditions. Furthermore, the proposed structure is expressed in a unique and broad manner, allowing us to explore certain specific cases previously addressed (see for example [4]). Here, we establish certain analytical criteria to validate the suggested novel existence results of hybrid Caputo-Hadamard fractional differential problems. The method used to accomplish the goals is based on Dhage's fixed point result.

The following structure is used to arrange the current manuscript. In Section 2, we collected the basic concepts regarding Caputo-Hadamard fractional operators and some requisite notions which are related to multi-valued mappings. In Section 3, we present the existence of a solution to both problems utilizing Dhage's analytical criteria. Section 4 presents two numerical examples to demonstrate the applicability of our analytical conclusions. The concluding remarks are addressed in Section 5.

\section{Preliminaries}

In this section, we present some basic definitions and notations utilized in the proof of the main results. Let $w \geq 0$ and suppose that the function $s:(a, b) \rightarrow \Re$ is integrable. Fractional Caputo-Hadamard integral of a function $s \in C((a, b), \Re)$ of order $w$ is presented by ${ }^{C H} \Im_{a^{+}}^{0}(s(\ell))=s(\ell)$ and

$$
{ }^{C H} \Im_{a^{+}}^{w}(s(\ell))=\frac{1}{\Gamma(w)} \int_{a}^{\ell}\left(\ln \frac{\ell}{x}\right)^{w-1} s(x) \frac{d x}{x}
$$


whenever the RHS integral exists. Keep in mind that for each $w_{1}, w_{2} \in \Re^{+}$, the following equality

$$
\mathrm{CH}_{\Im_{a^{+}}}^{w_{1}} \mathrm{CH}_{\Im_{a^{+}}}^{w_{2}}(s(\ell))={ }^{\mathrm{CH}} \Im_{a^{+}}^{w_{1}+w_{2}}(s(\ell))
$$

holds true and

$$
{ }^{C H} \Im_{a^{+}}^{w_{1}}\left(\ln \frac{\ell}{a}\right)^{w_{2}}=\frac{\Gamma\left(w_{2}+1\right)}{\Gamma\left(w_{1}+w_{2}+1\right)}\left(\ln \frac{\ell}{a}\right)^{w_{1}+w_{2}}
$$

for $\ell>a$. It is noticeable that for $w_{2}=0$ [2], the above equation reduces to

$$
\mathrm{CH}_{\Im_{a^{+}}}^{w_{1}} 1=\frac{1}{\Gamma\left(w_{1}+1\right)}\left(\ln \frac{\ell}{a}\right)^{w_{1}}, \forall \ell>a .
$$

Now, assume $\beta=[w]+1$ or $w \in[\beta-1, \beta)$. For a real-valued continuous function $s$ defined on $(a, b)$, the fractional Caputo-Hadamard derivative of order $w$ is defined as follows:

$$
{ }^{C} H_{\mathfrak{D}_{a^{+}}^{w}}(s(\ell))=\frac{1}{\Gamma(\beta-w)}\left(\ell \frac{d}{d \ell}\right)^{\beta} \int_{a}^{\ell}\left(\ln \frac{\ell}{x}\right)^{(\beta-w-1)} s(x) \frac{d x}{x}
$$

whenever the RHS integral exists [17]. Assuming $s \in A C_{\Re}^{m}([a, b])$ and $\beta-1<w \leq \beta$, a general solution for the Caputo-Hadamard differential equation ${ }^{C H} \mathfrak{D}_{a^{+}}^{w}(s(\ell))=0$ is of the form $s(\ell)=\sum_{k=0}^{\beta-1} d_{k}\left(\ln \frac{\ell}{a}\right)^{k}$, and we have

$$
{ }^{C H} \Im_{a^{+}}^{w}{ }^{C H} \mathfrak{D}_{a^{+}}^{w}(s(\ell))=s(\ell)+d_{0}+d_{1}\left(\ln \frac{\ell}{a}\right)+d_{2}\left(\ln \frac{\ell}{a}\right)^{2}+\ldots+d_{\beta-1}\left(\ln \frac{\ell}{a}\right)^{\beta-1},
$$

where, $d_{0}, d_{1}, \ldots, d_{\beta-1}$ are real constants and $\beta=[w]+1$ [23].

In the sequel, we assume $\left(\mathcal{N},\|\cdot\|_{\mathcal{N}}\right)$ a normed space, collection of all subsets of $\mathcal{N}$, all compact subsets of $\mathcal{N}$, all convex subsets of $\mathcal{N}$, all bounded subsets of $\mathcal{N}$, all closed subsets of $\mathcal{N}$ by $\mathcal{I}(\mathcal{N}), \mathcal{I}_{c m p}(\mathcal{N}), \mathcal{I}_{c v x}(\mathcal{N}), \mathcal{I}_{\text {bnd }}(\mathcal{N}), \mathcal{I}_{c l s}(\mathcal{N})$, respectively. Moreover, the following notions from $[24,25]$ are essential:

- If for each $\sigma \in \mathcal{N}$, the set $\omega(\sigma)$ has convex values, then we say that the set-valued map $\omega$ is convex.

- The set-valued map $\omega$ is said to be an upper semi-continuous map if for every $\sigma^{*} \in$ $\mathcal{N}, \omega\left(\sigma^{*}\right)$ belongs to $\mathcal{I}_{c l s}(\mathcal{N})$ and for every open set $\mathcal{O}$ with $\omega\left(\sigma^{*}\right) \subset \mathcal{O}$, there is a neighborhood $\mathcal{U}_{0}^{*}$ of $\sigma^{*}$ such that $\mathscr{\omega}\left(\mathcal{U}_{0}^{*}\right) \subset \mathcal{O}$.

- The set-valued map $\omega: \mathcal{N} \rightarrow \mathcal{I}(\mathcal{N})$ has a fixed point $\sigma^{*} \in \mathcal{N}$ if $\sigma^{*} \in \mathscr{\omega}\left(\sigma^{*}\right)$. The collection of all fixed points of $\omega$ is represented by $\curlyvee(\mathfrak{\omega})$.

- $\quad$ Assume $\left(\mathcal{N}, d_{\mathcal{N}}\right)$ to be a metric space. For each $F_{1}, F_{2} \in \mathcal{I}(\mathcal{N})$, the Pompeiu-Hausdorff metric $P H_{d}: \mathcal{I}(\mathcal{N}) \times \mathcal{I}(\mathcal{N}) \rightarrow \Re \cup\{\infty\}$ is defined as

$$
P H_{d}\left(F_{1}, F_{2}\right)=\max \left\{\sup _{a_{1} \in F_{1}} d_{N}\left(a_{1}, F_{2}\right), \sup _{a_{2} \in F_{2}} d_{N}\left(F_{1}, a_{2}\right)\right\},
$$

where, $d_{\mathcal{N}}\left(F_{1}, a_{2}\right)=\inf _{a_{1} \in F_{1}} d_{\mathcal{N}}\left(a_{1}, a_{2}\right)$ and $d_{\mathcal{N}}\left(a_{1}, F_{2}\right)=\inf _{a_{2} \in F_{2}} d_{\mathcal{N}}\left(a_{1}, a_{2}\right)$.

- The set-valued map $\mathscr{\omega}: \mathcal{N} \rightarrow \mathcal{I}_{c l s}(\mathcal{N})$ is said to be Lipschitzian if $P H_{d_{N}}\left(\omega\left(\sigma_{1}\right), \omega\left(\sigma_{2}\right)\right) \leq$ $m^{*} d_{\mathcal{N}}\left(\sigma_{1}, \sigma_{2}\right)$ holds for every $\sigma_{1}, \sigma_{2} \in \mathcal{N}$, where $m^{*}>0$ is a Lipschitz constant. If $0<m^{*}<1$, then we say that the Lipschitz map is a contractive map.

- We say that $\mathfrak{\omega}:[1, e] \rightarrow \mathcal{I}_{c l s}(\Re)$ is measurable if the function $\ell \rightarrow d_{\mathcal{N}}(r, \mathcal{\omega}(\ell))$ is measurable $\forall r \in \Re$.

- The graph of $\mathscr{\omega}: \mathcal{N} \rightarrow \mathcal{I}_{\text {cls }}(\mathcal{T})$ is defined by $\operatorname{Graph}(\mathscr{\omega})=\left\{\left(\sigma_{1}, \sigma_{2}\right) \in \mathcal{N} \times \mathcal{T}: s^{*} \in\right.$ $\omega(\sigma)\}$. It is noticeable that the graph of $\omega$ is said to be closed if for every arbitrary sequence $\left\{s_{n}\right\}_{n \geq 1} \in \mathcal{N}$ and $\left\{\sigma_{n}\right\}_{n \geq 1} \in \mathcal{T}$ with $s_{n} \rightarrow s_{0}, \sigma_{n} \rightarrow \sigma_{0}$ and $\sigma_{n} \in \mathcal{W}\left(s_{n}\right)$, we obtain $\sigma_{0} \in \mathscr{\omega}\left(s_{0}\right)$. If $\mathscr{\omega}: \mathcal{N} \rightarrow \mathcal{I}_{\text {cls }}(\mathcal{T})$ is an upper semi-continuous map, then $\operatorname{Graph}(\omega) \subseteq \mathcal{N} \times \mathcal{T}$ is a closed set. 
- A set-valued map $\mathscr{\omega}$ is completely continuous operator if the $\mathscr{\omega}(\mathcal{M})$ is relatively compact $\forall \mathcal{M} \in \mathcal{I}_{\text {bnd }}(\mathcal{N})$. Furthermore, we assume that by the complete continuity assumption, the map $\omega$ has a closed graph. Then, the multi-valued map $\omega$ is upper semi-continuous.

- The set-valued map $\mathscr{\omega}:[1, e] \times \Re \rightarrow \mathcal{I}(\Re)$ has a Caratheodory property if the function $\sigma \rightarrow \mathscr{\omega}(\ell, \sigma)$ is upper semi-continuous $\forall \ell \in[1, e]$ and the function $\ell \rightarrow \mathscr{\omega}(\ell, \sigma)$ is measurable for every $\sigma \in \Re$. Furthermore, A Caratheodory multi-valued mapping $\omega:[1, e] \times \Re \rightarrow \mathcal{I}(\Re)$ has $\mathcal{L}^{1}$-Caratheodory property if for every $s>0$ there exists $\theta_{S} \in \mathcal{L}_{\Re^{+}}^{1}([1, e])$ provided that

$$
\|\mathfrak{\omega}(\ell, \sigma)\|=\sup _{\ell \in[1, e]}\{|p|: p \in \mathfrak{W}(\ell, \sigma)\} \leq \theta_{\mathcal{S}}(\ell)
$$

for all $\ell \in[1, e]$ and for every $|\sigma| \leq s$.

- The selections of $\omega$ at $\sigma \in C_{\Re}([1, e])$ are represented by

$$
(\mathfrak{S E L})_{\mathscr{\omega}, \sigma}:=\left\{v \in \mathcal{L}_{\Re^{+}}^{1}([1, e]): v(\ell) \in \mathcal{W}(\ell, \sigma(\ell)), \forall \ell \in[1, e]\right\} .
$$

It is known that $(\mathfrak{S E L})_{\omega, \sigma} \neq \varphi$ for all $\sigma \in C_{\mathcal{N}}([1, e])$ whenever $\operatorname{dim} \mathcal{N}<\infty$.

We now state fixed point results due to Dhage and a closed graph theorem, which will be used to prove the existence results of our proposed problems.

Theorem 1 ([26]). Assume a Banach space $\mathcal{N}$. For almost all $\lambda \in \Re^{+}$, assume an open ball $v_{\lambda}(0)$ and its closure $\bar{v}_{\lambda}(0)$. Suppose that $\theta_{1}: \mathcal{N} \rightarrow \mathcal{N}$ and $\theta_{2}: \bar{v}_{\lambda}(0) \rightarrow \mathcal{N}$ are two operators that meet the properties listed

1. $\theta_{1}$ is a Lipschitzian map so that $m^{*}$ is a Lipschitz constant;

2. $\theta_{2}$ is completely continuous;

3. $m^{*} \triangle^{*}<1$, where $\triangle^{*}=\left\|\theta_{2}\left(\bar{v}_{\lambda}(0)\right)\right\|_{\mathcal{N}}=\sup \left\{\left\|\theta_{2} l\right\|_{\mathcal{N}}: l \in \bar{v}_{\lambda}(0)\right\}$.

Then either

(i) The operator equation $\theta_{1} \ell \theta_{2} \ell=\ell$ has a solution contained in $\bar{v}_{\lambda}(0)$ or;

(ii) There exists $\vartheta^{*} \in \mathcal{N}$ with $\left\|\vartheta^{*}\right\|_{\mathcal{N}}=\lambda$ so that $w_{0} \theta_{1} \vartheta^{*} \theta_{2} \vartheta^{*}=\vartheta^{*}$ for some $w_{0} \in(0,1)$.

Theorem 2 ([27]). Assume a separable Banach space $\mathcal{N}$, an $\mathcal{L}^{1}$-Caratheodory multi-valued function $\omega:[1, e] \times \mathcal{N} \rightarrow \mathcal{I}_{\text {cmp,cvx }}(\mathcal{N})$ and a linear continuous function $\Lambda: \mathcal{L}_{\mathcal{N}}^{1}([1, e]) \rightarrow$ $C_{\mathcal{N}}([1, e])$. Then, $\Lambda o\left((\mathfrak{S E L})_{\mathscr{\omega}}: C_{\mathcal{N}}([1, e]) \rightarrow \mathcal{I}_{\text {cmp,cvx }}\left(C_{\mathcal{N}}([1, e])\right)\right.$ is an operator belonging to $C_{\mathcal{N}}([1, e]) \times C_{\mathcal{N}}([1, e])$ defined by $\sigma \rightarrow\left(\lambda \circ(\mathfrak{S E E})_{\omega}\right)(\sigma)=\lambda\left((\mathfrak{S E L})_{\omega, \sigma}\right)$ and has a closed graph property.

Theorem 3 ([28]). Assume the Banach space $\mathcal{N}$. Consider that there is a single-valued map $\theta_{1}: \mathcal{N} \rightarrow \mathcal{N}$ and a multi-valued map $\theta_{2}: \mathcal{N} \rightarrow \mathcal{I}_{\text {cmp,cvx }}(\mathcal{N})$ satisfying the following properties:

1. $\theta_{1}$ is a Lipschitzian map so that $m^{*}$ is a Lipschitz constant;

2. $\quad \theta_{2}$ has compactness and an upper-semi continuity property;

3. $2 m^{*} \triangle^{*}<1$ with $\triangle^{*}=\left\|\theta_{2}(\mathcal{N})\right\|$.

Then either

(i) there exists a solution contained in $\mathcal{N}$ for the inclusion $\ell \in \theta_{1} \ell \theta_{2} l$ or;

(ii) the set $O=\left\{\vartheta^{*} \in \mathcal{N} \mid w_{0} \vartheta^{*} \in \theta_{1} \vartheta^{*} \theta_{2} \vartheta^{*}, w_{0}>1\right\}$ is an unbounded set.

\section{Main Results}

Here, we assume $\mathcal{N}=C_{\Re}([1, e])$ to be a Banach space with the standard norm, $\|s\|_{\mathcal{N}}=\sup \{|s(\ell)|: \ell \in[1, e]\}$. 
Lemma 1. Consider $\gamma \in \mathcal{N}$. The hybrid CH-FBVP,

$$
C H \mathfrak{D}_{1^{+}}^{w}\left(\frac{s(\ell)}{g(\ell, s(\ell))}\right)+\gamma(\ell)=0, \quad \ell \in[1, e], w \in(1,2],
$$

supplemented with the three-point hybrid Caputo-Hadamard terminal conditions

$$
\begin{aligned}
& \left.\mathfrak{D}\left(\frac{s(\ell)}{g(\ell, s(\ell))}\right)\right|_{\ell=1}=0 \\
& \left.\tau^{C H} \mathfrak{D}_{1^{+}}^{w-1}\left(\frac{s(\ell)}{g(\ell, s(\ell))}\right)\right|_{\ell=e}+\left.\left(\frac{s(\ell)}{g(\ell, s(\ell))}\right)\right|_{\ell=\zeta}=0,
\end{aligned}
$$

has a solution $s_{0}$, iff $s_{0}$ is a solution for the Caputo-Hadamard integral equation,

$$
\begin{aligned}
s(\ell) & =g(\ell, s(\ell))\left[-\frac{1}{\Gamma(w)} \int_{1}^{\ell}\left(\ln \frac{\ell}{x}\right)^{w-1} \gamma(x) \frac{d x}{x}+\tau \int_{1}^{e} \gamma(x) \frac{d x}{x}\right. \\
& \left.+\frac{1}{\Gamma(w)} \int_{1}^{\zeta}\left(\ln \frac{\zeta}{x}\right)^{w-1} \gamma(x) \frac{d x}{x}\right] .
\end{aligned}
$$

Proof. Assume that hybrid Equation (5) has a solution $s_{0}$. Then, by utilizing the equality, $\frac{s_{0}(\ell)}{g\left(\ell, s_{0}(\ell)\right)}=-{ }^{C H} \Im_{1^{+}}^{w} \gamma(\ell)+c_{0}+c_{1} \ln (\ell)$, where $c_{0}$ and $c_{1} \in \Re$, the homogeneous Equation (5) has general solution given below:

$$
s_{0}(\ell)=g\left(\ell, s_{0}(\ell)\right)\left[-\frac{1}{\Gamma(w)} \int_{1}^{\ell}\left(\ln \frac{\ell}{x}\right)^{w-1} \gamma(x) \frac{d x}{x}+c_{0}+c_{1} \ln (\ell)\right] .
$$

Applying ordinary derivative $\mathfrak{D}=\frac{d}{d \ell}$ on (8), we get

$$
\begin{aligned}
\mathfrak{D}\left(\frac{s_{0}}{g\left(\ell, s_{0}(\ell)\right)}\right) & =-\frac{1}{\Gamma(w)} \int_{1}^{\ell}(w-1)\left(\ln \frac{\ell}{x}\right)^{w-2} \frac{\gamma(x)}{\ell} d x+\frac{1}{\ell} c_{1} \\
& =-\frac{1}{\Gamma(w-1)} \int_{1}^{\ell}\left(\ln \frac{\ell}{x}\right)^{w-2} \frac{\gamma(x)}{\ell} d x+\frac{1}{\ell} c_{1} .
\end{aligned}
$$

Using the first condition given in (6), we get $c_{1}=0$. Now, by applying ${ }^{C H_{1}} \mathfrak{D}_{1^{+}}^{w-1}$ on both sides of (8), we have

$$
\begin{aligned}
C_{\mathfrak{D}_{1}^{+}}^{w-1}\left(\frac{s_{0}(\ell)}{g\left(\ell, s_{0}(\ell)\right)}\right) & =-\int_{1}^{\ell} \gamma(x) \frac{d x}{x} . \\
\left.\Rightarrow \tau^{C H} \mathfrak{D}_{1^{+}}^{w-1}\left(\frac{s_{0}(\ell)}{g\left(\ell, s_{0}(\ell)\right)}\right)\right|_{\ell=e} & =-\tau \int_{1}^{e} \gamma(x) \frac{d x}{x} . \\
\left.\Rightarrow\left(\frac{s_{0}(\ell)}{g\left(\ell, s_{0}(\ell)\right)}\right)\right|_{\ell=\zeta} & =-\frac{1}{\Gamma(w)} \int_{1}^{\zeta}\left(\ln \frac{\zeta}{x}\right)^{w-1} \gamma(x) \frac{d x}{x}+c_{0} .
\end{aligned}
$$


Using the second condition given in (6), we obtain

$$
c_{0}=\tau \int_{1}^{e} \gamma(x) \frac{d x}{x}+\frac{1}{\Gamma(w)} \int_{1}^{\zeta}\left(\ln \frac{\zeta}{x}\right)^{w-1} \gamma(x) \frac{d x}{x}
$$

Now, by using the values of $c_{0}$ and $c_{1}$ in (8), we obtain

$$
\begin{aligned}
& s_{0}(\ell)=g\left(\ell, s_{0}(\ell)\right)\left[-\frac{1}{\Gamma(w)} \int_{1}^{\ell}\left(\ln \frac{\ell}{x}\right)^{w-1} \gamma(x) \frac{d x}{x}+\tau \int_{1}^{e} \gamma(x) \frac{d x}{x}\right. \\
& \left.+\frac{1}{\Gamma(w)} \int_{1}^{\zeta}\left(\ln \frac{\zeta}{x}\right)^{w-1} \gamma(x) \frac{d x}{x}\right] .
\end{aligned}
$$

This implies that the fractional integral Equation (7) has a unique solution $s_{0}$. In the reverse order, it is easy to see that if the fractional integral Equation (7) has a solution $s_{0}$ then $s_{0}$ satisfies the fractional hybrid CH-FBVP (5) and (6).

Now, we provide the existence result for the solution of problem (1) and (2).

Theorem 4. Suppose that $g \in C([1, e] \times \Re, \Re \backslash\{0\})$ and $\eta \in C([1, e] \times \Re, \Re)$ and

$\left(\mathcal{S}_{1}\right)$ there exists bounded function $k:[1, e] \rightarrow \Re^{+}$such that $\forall s_{1}, s_{2} \in \Re$, we have

$$
\left|g\left(\ell, s_{1}\right)-g\left(\ell, s_{2}\right)\right| \leq k(\ell)\left|s_{1}(\ell)-s_{2}(\ell)\right|
$$

for all $s_{1}, s_{2} \in \Re$ and $\ell \in[1, e]$,

$\left(\mathcal{S}_{2}\right)$ there exists a continuous increasing map $\mathcal{M}:[0, \infty) \rightarrow(0, \infty)$ and a continuous map $h:[1, e] \rightarrow \Re^{+}$such that

$$
|\eta(\ell, s)| \leq h(\ell) \mathcal{M}(\|s\|), \forall \ell \in[1, e] \text { and } s \in \Re,
$$

$\left(\mathcal{S}_{3}\right)$ there exits a number $\epsilon \in \Re^{+}$such that

$$
\epsilon>\frac{G^{*} \Delta^{*} H^{*} \mathcal{M}(\|s\|)}{1-k^{*} \Delta^{*} H^{*} \mathcal{M}(\|s\|)}
$$

where, $G^{*}=\sup _{\ell \in[1, e]}|g(\ell, 0)|, H^{*}=\sup _{\ell \in[1, e]}|h(\ell)|, k^{*}=\sup _{\ell \in[1, e]}|k(\ell)|$ and

$$
\Delta^{*}=\frac{1}{\Gamma(w+1)}+\frac{(\ln \zeta)^{w}}{\Gamma(w+1)}+\tau
$$

If $k^{*} \Delta^{*} H^{*} \mathcal{M}(\|s\|)<1$, then the hybrid CH-FBVP, (1)-(2) has unique solution.

Proof. Assume a closed ball $\bar{\mu}_{\epsilon}(0):=\left\{s \in \mathcal{N}:\|s\|_{\mathcal{N}} \leq \epsilon\right\}$ in the Banach space $\mathcal{N}$, where $\epsilon$ meets the inequality (10). By utilizing fractional integral Equation (7) and Lemma 1, we define two operators $\mathcal{A}_{1}, \mathcal{A}_{2}: \bar{\mu}_{\epsilon}(0) \rightarrow \mathcal{N}$ by $\left(\mathcal{A}_{1} s\right)(\ell)=g(\ell, s(\ell))$ and

$$
\begin{aligned}
\left(\mathcal{A}_{2} s\right)(\ell) & =-\int_{1}^{\ell} \frac{1}{\Gamma(w)}\left(\ln \frac{\ell}{x}\right)^{w-1} \eta(x, s(x)) \frac{d x}{x}+\tau \int_{1}^{e} \eta(x, s(x)) \frac{d x}{x} \\
& +\int_{1}^{\zeta} \frac{1}{\Gamma(w)}\left(\ln \frac{\zeta}{x}\right)^{w-1} \eta(x, s(x)) \frac{d x}{x} .
\end{aligned}
$$

Obviously, $s \in \mathcal{N}$ is a solution for the fractional hybrid BVP (1) and (2) and satisfies the operator equation $\mathcal{A}_{1} s \mathcal{A}_{2} s=s$. By utilizing the conditions of Theorem 1 , we show that 
a solution exists. First, we show that the operator $\mathcal{A}_{1}$ is a Lipschitzian map on normed algebra $\mathcal{N}$ having constant $\left.k^{*}=\sup _{[1, e]} \mid k(\ell)\right) \mid$.

Let $s_{1}, s_{2} \in \mathcal{N}$. By using assumption $\left(\mathcal{S}_{1}\right)$, we get

$$
\left|\left(\mathcal{A}_{1} s_{1}\right)(\ell)-\left(\mathcal{A}_{1} s_{2}\right)(\ell)\right|=\left|g\left(\ell, s_{1}(\ell)\right)-g\left(\ell, s_{2}(\ell)\right)\right| \leq k(\ell)\left|s_{1}(\ell)-s_{2}(\ell)\right|
$$

for all $s_{1}, s_{2} \in \bar{\mu}_{\epsilon}(0)$. This means that $\mathcal{A}_{1}$ is a Lipschitzian map on $\bar{\mu}_{\epsilon}(0)$ having a Lipschitz constant $k^{*}$. Now, we show the complete continuity of the operator $\mathcal{A}_{2}$ on $\bar{\mu}_{\epsilon}(0)$. Firstly, it is required to check that the map $\mathcal{A}_{2}$ is continuous on $\bar{\mu}_{\epsilon}(0)$. Assume $\left\{s_{n}\right\}$ is a sequence in the closed ball $\bar{\mu}_{\epsilon}(0)$ with $s_{n} \rightarrow s$, where $s \in \bar{\mu}_{\epsilon}(0)$.

As we know $\eta$ is continuous on $[1, e] \times \Re$, we conclude that $\lim _{n \rightarrow \infty} \eta\left(\ell, s_{n}(\ell)\right)=$ $\eta(\ell, s(\ell))$. With the help of the Lebesgue dominated convergence theorem, we get

$$
\begin{aligned}
\lim _{n \rightarrow \infty}\left(\mathcal{A}_{2} s_{n}\right)(\ell) & =-\int_{1}^{\ell} \frac{1}{\Gamma(w)}\left(\ln \frac{\ell}{x}\right)^{w-1} \lim _{n \rightarrow \infty} \eta\left(x, s_{n}(x)\right) \frac{d x}{x} \\
& +\tau \int_{1}^{e} \lim _{n \rightarrow \infty} \eta\left(x, s_{n}(x)\right) \frac{d x}{x} \\
& +\int_{1}^{\zeta} \frac{1}{\Gamma(w)}\left(\ln \frac{\zeta}{x}\right)^{w-1} \lim _{n \rightarrow \infty} \eta\left(x, s_{n}(x)\right) \frac{d x}{x} \\
& =-\int_{1}^{\ell} \frac{1}{\Gamma(w)}\left(\ln \frac{\ell}{x}\right)^{w-1} \lim _{n \rightarrow \infty} \eta(x, s(x)) \frac{d x}{x} \\
& +\tau \int_{1}^{e} \lim _{n \rightarrow \infty} \eta(x, s(x)) \frac{d x}{x} \\
& +\int_{1}^{\zeta} \frac{1}{\Gamma(w)}\left(\ln \frac{\zeta}{x}\right)^{w-1} \lim _{n \rightarrow \infty} \eta(x, s(x)) \frac{d x}{x} \\
& =\left(\mathcal{A}_{2} s\right)(\ell), \forall \ell \in[1, e] .
\end{aligned}
$$

Thus, $\mathcal{A}_{2} s_{n} \rightarrow \mathcal{A}_{2} s$ and so $\mathcal{A}_{2}$ is a continuous operator on $\bar{\mu}_{\epsilon}(0)$. Now, we check the uniform boundedness of the operator $\mathcal{A}_{2}$ on $\bar{\mu}_{\epsilon}(0)$. By assumption $\left(\mathcal{S}_{1}\right)$, we get

$$
\begin{aligned}
\left|\left(\mathcal{A}_{2} s\right)(\ell)\right| & =\int_{1}^{\ell} \frac{1}{\Gamma(w)}\left(\ln \frac{\ell}{x}\right)^{w-1} \lim _{n \rightarrow \infty}|\eta(x, s(x))| \frac{d x}{x}+\tau \int_{1}^{e} \lim _{n \rightarrow \infty}|\eta(x, s(x))| \frac{d x}{x} \\
& +\int_{1}^{\zeta} \frac{1}{\Gamma(w)}\left(\ln \frac{\zeta}{x}\right)^{w-1} \lim _{n \rightarrow \infty}|\eta(x, s(x))| \frac{d x}{x} \\
& \leq \frac{(\ln \ell)^{w}}{\Gamma(w+1)} h(x) \mathcal{M}(\|s\|)+\tau h(x) \mathcal{M}(\|s\|)+\frac{(\ln \zeta)^{w}}{\Gamma(w+1)} h(x) \mathcal{M}(\|s\|) \\
& =h(x) \mathcal{M}(\|s\|)\left[\frac{(\ln \ell)^{w}}{\Gamma(w+1)}+\tau+\frac{(\ln \zeta)^{w}}{\Gamma(w+1)}\right],
\end{aligned}
$$

$\forall \ell \in[1, e]$ and $s \in \bar{\mu}_{\epsilon}(0)$. By taking supremum over $[1, e]$, we have

$$
\left\|\mathcal{A}_{2} s\right\| \leq H^{*} \mathcal{M}(\|s\|) \triangle^{*},
$$


where, $\triangle^{*}$ is given in (11). This implies that in normed algebra $\mathcal{N}$, the set $\mathcal{A}_{2}\left(\bar{\mu}_{\epsilon}(0)\right)$ is uniformly bounded. The equi-continuity of the operator $\mathcal{A}_{2}$ is now being explored. For this purpose, we suppose $\ell_{1}, \ell_{2} \in[1, e]$ with $\ell_{1}<\ell_{2}$. Then, we have

$$
\begin{aligned}
\left|\left(\mathcal{A}_{2} s\right)\left(\ell_{2}\right)-\left(\mathcal{A}_{2} s\right)\left(\ell_{1}\right)\right| & =\mid \int_{1}^{\ell_{2}} \frac{1}{\Gamma(w)}\left(\ln \frac{\ell_{2}}{x}\right)^{w-1} \eta(x, s(x)) \frac{d x}{x} \\
& -\int_{1}^{\ell_{1}} \frac{1}{\Gamma(w)}\left(\ln \frac{\ell_{1}}{x}\right)^{w-1} \eta(x, s(x)) \frac{d x}{x} \mid \\
& \leq H^{*} \mathcal{M}(\|s\|)\left[\int_{1}^{\ell_{1}}\left(\frac{1}{\Gamma(w)}\left(\ln \frac{\ell_{2}}{x}\right)^{w-1}-\frac{1}{\Gamma(w)}\left(\ln \frac{\ell_{1}}{x}\right)^{w-1}\right) \frac{d x}{x}\right. \\
& \left.+\int_{\ell_{1}}^{\ell_{2}} \frac{1}{\Gamma(w)}\left(\ln \frac{\ell_{2}}{x}\right)^{w-1} \frac{d x}{x}\right] .
\end{aligned}
$$

It is noticeable that the RHS of the above inequality approaches zero independent of $s \in \bar{\mu}_{\epsilon}(0)$ as $\ell_{1} \rightarrow \ell_{2}$. Thus, the operator $\mathcal{A}_{2}$ is equi-continuous. By utilizing the ArzelaAscoli theorem, it is inferred that $\mathcal{A}_{2}$ is completely continuous on $s \in \bar{\mu}_{\epsilon}(0)$.

Now, by utilizing $\left(\mathcal{S}_{3}\right)$, we have

$$
\begin{aligned}
M_{0}^{*} & =\left\|\mathcal{A}_{2}\left(\bar{\mu}_{\epsilon}(0)\right)\right\|_{\mathcal{N}}=\sup \left\{\left|\mathcal{A}_{2} s\right|: s \in \bar{\mu}_{\epsilon}(0)\right\} \\
& =H^{*} \mathcal{M}(\|s\|)\left[\frac{1}{\Gamma(w+1)}+\tau+\frac{(\ln \zeta)^{w}}{\Gamma(w+1)}\right] \\
& =H^{*} \mathcal{M}(\|s\|) \triangle^{*}
\end{aligned}
$$

Setting $l^{*}=k^{*}$, we get $M_{0}^{*} l^{*}<1$. So, one of the condition (i) or (ii) in Theorem 1 is satisfied. For any $v \in(0,1)$, assume that $s$ satisfies the operator equation, $s=v \mathcal{A}_{1} \mathcal{A}_{2} s$ so that $\|s\|=\epsilon$ and we have

$$
\begin{aligned}
|s(\ell)| & =v\left|\left(\mathcal{A}_{1} s\right)(\ell)\right|\left|\left(\mathcal{A}_{2} s\right)(\ell)\right|=v|g(\ell, s(\ell))| \\
& \times \mid-\int_{1}^{\ell} \frac{1}{\Gamma(w)}\left(\ln \frac{\ell}{x}\right)^{w-1} \eta(x, s(x)) \frac{d x}{x} \\
& +\tau \int_{1}^{e} \eta(x, s(x)) \frac{d x}{x} \int_{1}^{\zeta} \frac{1}{\Gamma(w)}\left(\ln \frac{\zeta}{x}\right)^{w-1} \eta(x, s(x)) \frac{d x}{x} \mid \\
& \leq(\mid g(\ell, s(\ell))-g(\ell, 0))|+| g(\ell, 0) \mid) \\
& \times\left(\int_{1}^{\ell} \frac{1}{\Gamma(w)}\left(\ln \frac{\ell}{x}\right)^{w-1}|\eta(x, s(x))| \frac{d x}{x}\right. \\
& \left.+\tau \int_{1}^{e}|\eta(x, s(x))| \frac{d x}{x} \int_{1}^{\zeta} \frac{1}{\Gamma(w)}\left(\ln \frac{\zeta}{x}\right)^{w-1}|\eta(x, s(x))| \frac{d x}{x}\right) \\
& \leq\left(k(\ell)|s(\ell)|+G^{*}\right) \triangle^{*} H^{*} \mathcal{M}(\|s\|) \\
& \leq\left(k^{*}\|s\|+G^{*}\right) \triangle^{*} H^{*} \mathcal{M}(\|s\|) .
\end{aligned}
$$

So $\epsilon \leq \frac{G^{*} \triangle^{*} H^{*} \mathcal{M}(\|s\|)}{1-k^{*} \triangle^{*} H^{*} \mathcal{M}(\|s\|)}$ which is inconsistent with (10). This implies that the condition (ii) of Theorem 1 is not possible. Hence, the condition $(i)$ in Theorem 1 is satisfied and the fractional hybrid problem (1) and (2) has a solution. 
From the above theorem, we conclude that the solution of the hybrid Caputo-Hadamard fractional differential equation exists provided that the stated conditions hold.

Now, we provide our main results related to hybrid CH-FIBVP for the thermostat model (3) and (4).

Definition 1. We call the function $s \in A C_{\Re}([1, e])$ a solution set for hybrid $C H-F I B V P,(3)$ and (4) whenever there exists an integrable function $v \in \mathcal{L}^{1}([1, e], \Re)$ with $v(\ell) \in \omega(\ell, s(\ell)) \forall \ell \in[1, e]$, such that

$$
\left.\mathfrak{D}\left(\frac{s(\ell)}{g(\ell, s(\ell))}\right)\right|_{\ell=1}=0,\left.\quad \tau^{C H} \mathfrak{D}_{1^{+}}^{w-1}\left(\frac{s(\ell)}{g(\ell, s(\ell))}\right)\right|_{\ell=e}+\left.\left(\frac{s(\ell)}{g(\ell, s(\ell))}\right)\right|_{\ell=\zeta}=0
$$

and

$$
\begin{aligned}
s(\ell) & =g(\ell, s(\ell))\left[-\frac{1}{\Gamma(w)} \int_{1}^{\ell}\left(\ln \frac{\ell}{x}\right)^{w-1} v(x) \frac{d x}{x}+\tau \int_{1}^{e} v(x) \frac{d x}{x}\right. \\
& \left.+\frac{1}{\Gamma(w)} \int_{1}^{\zeta}\left(\ln \frac{\zeta}{x}\right)^{w-1} v(x) \frac{d x}{x}\right] .
\end{aligned}
$$

Theorem 5. Assume that

$\left(\mathcal{S}_{4}\right)$ there is a bounded function $k:[1, e] \rightarrow \Re^{+}$such that $\forall s_{1}, s_{2} \in \Re$ and $\ell \in[1, e]$, we get

$$
\left|g\left(\ell, s_{1}(\ell)\right)-g\left(\ell, s_{2}(\ell)\right)\right| \leq k(\ell)\left|s_{1}(\ell)-s_{2}(\ell)\right|,
$$

$\left(\mathcal{S}_{5}\right)$ the compact and convex-valued multi-function $\omega:[1, e] \times \Re \rightarrow \mathcal{I}_{c p, c v}(\Re)$ is $\mathcal{L}^{1}$ Caratheodary,

$\left(\mathcal{S}_{6}\right)$ there exits a positive function $p \in \mathcal{L}^{1}\left([1, e], \Re^{+}\right)$such that

$$
\|\mathfrak{\omega}(\ell, s)\|=\sup \{|v|: v \in \mathfrak{W}(\ell, s(\ell))\} \leq p(\ell)
$$

$\forall s \in \Re$, almost $\forall \ell \in[1, e]$ and $\|p\|_{\mathcal{L}^{1}}=\int_{1}^{e}|p(x)| d x$,

$\left(\mathcal{S}_{7}\right)$ there is a number $\tilde{\epsilon} \in \Re^{+}$such that

$$
\tilde{\epsilon}>\frac{G^{*} \Delta^{*}\|p\|_{\mathcal{L}^{1}}}{1-k^{*} \Delta^{*}\|p\|_{\mathcal{L}^{1}}}
$$

where $G^{*}=\sup _{\ell \in[1, e]}|g(\ell, 0)|, k^{*}=\sup _{\ell \in[1, e]}|k(\ell)|$ and $\Delta^{*}$ is as given in (11). Then, hybrid CH-FIBVP (3) and (4) has at least one solution whenever $k^{*} \Delta^{*}\|p\|_{\mathcal{L}^{1}}<\frac{1}{2}$.

Proof. Consider an operator $\mathcal{K}: \mathcal{N} \rightarrow \mathcal{I}(\mathcal{N})$ defined by

$$
\mathcal{K}(s)=\left\{w \in \mathcal{N}: w(\ell)=k_{1}(\ell) \text { for all } 1 \leq \ell \leq e\right\},
$$

where

$$
\begin{aligned}
k_{1}(\ell) & =g(\ell, s(\ell))\left(-\frac{1}{\Gamma(w)} \int_{1}^{\ell}\left(\ln \frac{\ell}{x}\right)^{w-1} v(x) \frac{d x}{x}+\tau \int_{1}^{e} v(x) \frac{d x}{x}\right. \\
& \left.+\frac{1}{\Gamma(w)} \int_{1}^{\zeta}\left(\ln \frac{\zeta}{x}\right)^{w-1} v(x) \frac{d x}{x}\right), v \in(\mathfrak{S E L})_{\mathcal{W}, s} .
\end{aligned}
$$


Note that the solution for hybrid CH-FIBVP (3) and (4) is a fixed point of the map $\mathcal{K}$. Define a single-valued function, $\mathcal{A}_{1}: \mathcal{N} \rightarrow \mathcal{N}$ by $\left(\mathcal{A}_{1} s\right)(\ell)=g(\ell, s(\ell))$ and the set-valued $\operatorname{map}, \mathcal{A}_{2}: \mathcal{N} \rightarrow \mathcal{I}(\mathcal{N})$ by

$$
\left(\mathcal{A}_{2} s\right)(\ell)=\left\{\psi \in \mathcal{N}: \psi(\ell)=k_{2}(\ell) \text { for all } \ell \in[1, e]\right\}
$$

where

$$
\begin{aligned}
& k_{2}(\ell)=\left(-\frac{1}{\Gamma(w)} \int_{1}^{\ell}\left(\ln \frac{\ell}{x}\right)^{w-1} v(x) \frac{d x}{x}+\tau \int_{1}^{e} v(x) \frac{d x}{x}\right. \\
& \left.+\frac{1}{\Gamma(w)} \int_{1}^{\zeta}\left(\ln \frac{\zeta}{x}\right)^{w-1} v(x) \frac{d x}{x}\right), v \in(\mathfrak{S E L})_{\mathcal{O}, s} .
\end{aligned}
$$

Note that $\mathcal{K}(s)=\mathcal{A}_{1} s \mathcal{A}_{2}$ s. We prove that $\mathcal{A}_{1}$ and $\mathcal{A}_{2}$ satisfy the assumptions of Theorem 3. Using $\left(\mathcal{S}_{4}\right)$ and by a comparable conclusion in Theorem $4, \mathcal{A}_{1}$ is Lipschitz on $\mathcal{N}$. We can now see that the multi-valued function $\mathcal{A}_{2}$ has convex values. Assume $s_{1}, s_{2} \in \mathcal{A}_{2}$ s. Then, select $v_{1}, v_{2} \in(\mathfrak{S E L})_{\mathcal{W}, S}$ such that for $i=1,2$, we have

$$
\begin{aligned}
s_{i}(\ell) & =-\frac{1}{\Gamma(w)} \int_{1}^{\ell}\left(\ln \frac{\ell}{x}\right)^{w-1} v_{i}(x) \frac{d x}{x}+\tau \int_{1}^{e} v_{i}(x) \frac{d x}{x} \\
& +\frac{1}{\Gamma(w)} \int_{1}^{\zeta}\left(\ln \frac{\zeta}{x}\right)^{w-1} v_{i}(x) \frac{d x}{x}, \forall \ell \in[1, e] .
\end{aligned}
$$

For every constant $q \in(0,1)$, we get

$$
\begin{aligned}
q s_{1}(\ell)+(1-q) s_{2}(\ell) & =-\frac{1}{\Gamma(w)} \int_{1}^{\ell}\left(\ln \frac{\ell}{x}\right)^{w-1}\left[q v_{1}(x)+(1-q) v_{2}(x)\right] \frac{d x}{x} \\
& +\tau \int_{1}^{e}\left[q v_{1}(x)+(1-q) v_{2}(x)\right] \frac{d x}{x} \\
& +\frac{1}{\Gamma(w)} \int_{1}^{\zeta}\left(\ln \frac{\zeta}{x}\right)^{w-1}\left[q v_{1}(x)+(1-q) v_{2}(x)\right] \frac{d x}{x}
\end{aligned}
$$

$\forall \ell \in[1, e]$. As we know that $\mathfrak{\omega}$ is convex-valued, $(\mathfrak{S} \mathfrak{E} \mathfrak{L})_{\mathfrak{W}, S}$ has convex values and so $q v_{1}(\ell)+(1-q) v_{2}(\ell) \in(\mathfrak{S E L})_{\mathcal{O}, s} \forall \ell \in[1, e]$. So that $\mathcal{A}_{2} s$ is a convex set for all $s \in \mathcal{N}$. To check the complete continuity of the operator $\mathcal{A}_{2}$, we must verify that $\mathcal{A}_{2}(\mathcal{N})$ is uniformly bounded and an equi-continuous set. For this reason, we prove that $\mathcal{A}_{2}$ mapped all bounded sets into bounded subsets of the space $\mathcal{N}$. For a number $\epsilon^{*} \in \Re^{+}$, assume a bounded ball $v_{\epsilon}^{*}=\left\{s \in \mathcal{N}:\|s\|_{\mathcal{N}} \leq \epsilon^{*}\right\}$. For every $s \in v_{\epsilon}^{*}$ and $\psi \in \mathcal{A}_{2} s$, a function $v \in(\mathfrak{S E \mathfrak { L }})_{\mathcal{O}, \mathrm{S}}$ exists such that

$$
\begin{aligned}
\psi(\ell) & =-\frac{1}{\Gamma(w)} \int_{1}^{\ell}\left(\ln \frac{\ell}{x}\right)^{w-1} v(x) \frac{d x}{x}+\tau \int_{1}^{e} v(x) \frac{d x}{x} \\
& +\frac{1}{\Gamma(w)} \int_{1}^{\zeta}\left(\ln \frac{\zeta}{x}\right)^{w-1} v(x) \frac{d x}{x}, \forall \ell \in[1, e] .
\end{aligned}
$$


Then, we get

$$
\begin{aligned}
|\psi(\ell)| & \leq \frac{1}{\Gamma(w)} \int_{1}^{\ell}\left(\ln \frac{\ell}{x}\right)^{w-1}|v(x)| \frac{d x}{x}+\tau \int_{1}^{e}|v(x)| \frac{d x}{x} \\
& +\frac{1}{\Gamma(w)} \int_{1}^{\zeta}\left(\ln \frac{\zeta}{x}\right)^{w-1}|v(x)| \frac{d x}{x} \\
& \leq \frac{1}{\Gamma(w)} \int_{1}^{\ell}\left(\ln \frac{\ell}{x}\right)^{w-1} p(x) \frac{d x}{x}+\tau \int_{1}^{e} p(x) \frac{d x}{x} \\
& +\frac{1}{\Gamma(w)} \int_{1}^{\zeta}\left(\ln \frac{\zeta}{x}\right)^{w-1} p(x) \frac{d x}{x} \\
& \leq\left[\frac{1}{\Gamma(w+1)}+\frac{(\ln \zeta)^{w}}{\Gamma(w+1)}+\tau\right]\|p\|_{\mathcal{L}^{1}} \\
& =\triangle^{*}\|p\|_{\mathcal{L}^{1}}
\end{aligned}
$$

where, $\triangle^{*}$ is as given in (11). Thus, $|\psi(\ell)| \leq \triangle^{*}\|p\|_{\mathcal{L}^{1}}$ and this implies that $\mathcal{A}_{2}(\mathcal{N})$ is a uniformly bounded set. We now see how the operator $\mathcal{A}_{2}$ mapped bounded sets onto equi-continuous sets. Let $s \in v_{\epsilon}^{*}$ and $\psi \in \mathcal{A}_{2} s$. We select $v \in(\mathfrak{S} \mathfrak{E} \mathfrak{L})_{\omega, s}$ so that

$$
\begin{aligned}
\psi(\ell) & =-\frac{1}{\Gamma(w)} \int_{1}^{\ell}\left(\ln \frac{\ell}{x}\right)^{w-1} v(x) \frac{d x}{x}+\tau \int_{1}^{e} v(x) \frac{d x}{x} \\
& +\frac{1}{\Gamma(w)} \int_{1}^{\zeta}\left(\ln \frac{\zeta}{x}\right)^{w-1} v(x) \frac{d x}{x}, \quad \forall \ell \in[1, e] .
\end{aligned}
$$

For each $l_{1}, l_{2} \in[1, e]$ with $\ell_{1}<\ell_{2}$, we obtain

$$
\begin{aligned}
\left|\psi\left(\ell_{2}\right)-\psi\left(\ell_{1}\right)\right| & \leq\left|\frac{1}{\Gamma(w)} \int_{1}^{\ell_{2}}\left(\ln \frac{\ell_{2}}{x}\right)^{w-1} v(x) \frac{d x}{x}-\frac{1}{\Gamma(w)} \int_{1}^{\ell_{1}}\left(\ln \frac{\ell_{1}}{x}\right)^{w-1} v(x) \frac{d x}{x}\right| \\
& =\mid \frac{1}{\Gamma(w)} \int_{1}^{\ell_{1}}\left(\ln \frac{\ell_{2}}{x}\right)^{w-1} v(x) \frac{d x}{x}+\frac{1}{\Gamma(w)} \int_{\ell_{1}}^{\ell_{2}}\left(\ln \frac{\ell_{2}}{x}\right)^{w-1} v(x) \frac{d x}{x} \\
& -\frac{1}{\Gamma(w)} \int_{1}^{\ell_{1}}\left(\ln \frac{\ell_{1}}{x}\right)^{w-1} v(x) \frac{d x}{x} \mid \\
& \leq \int_{1}^{\ell_{1}}\left(\frac{\left[\left(\ln \frac{\ell_{2}}{x}\right)^{w-1}-\left(\ln \frac{\ell_{2}}{x}\right)^{w-1}\right]}{\Gamma(w)}\right) p(x) \frac{d x}{x} \\
& +\frac{1}{\Gamma(w)} \int_{\ell_{1}}^{\ell_{2}}\left(\ln \frac{l_{2}}{x}\right)^{w-1} p(x) \frac{d x}{x} .
\end{aligned}
$$

It is noticeable that the RHS of the above inequalities goes to 0 independent of $s \in v_{\epsilon}^{*}$ as $\ell_{1} \rightarrow \ell_{2}$. By utilizing the Arzela-Ascoli theorem, the operator $\mathcal{A}_{2}: C([1, e], \Re) \rightarrow$ $\mathcal{I}(C([1, e], \Re))$ has the complete continuity property. We can now check that $\mathcal{A}_{2}$ has a closed graph which means that due to the complete continuity of $\mathcal{A}_{2}$, the operator $\mathcal{A}_{2}$ is upper semi-continuous. In this way, we assume that $s_{n} \in v_{\epsilon}^{*}$ and $\psi_{n} \in \mathcal{A}_{2} s_{n}$ are such that 
$s_{n} \rightarrow s^{*}$ and $\psi_{n} \rightarrow \psi^{*}$. We claim that $\psi^{*} \in \mathcal{A}_{2} s^{*}$. For each $n \geq 1$ and $\psi_{n} \in \mathcal{A}_{2} s_{n}$, choose $v_{n} \in(\mathfrak{S E E} \mathfrak{L})_{\omega_{,} s_{n}}$ such that

$$
\begin{aligned}
\psi_{n}(\ell) & =-\frac{1}{\Gamma(w)} \int_{1}^{\ell}\left(\ln \frac{\ell}{x}\right)^{w-1} v_{n}(x) \frac{d x}{x}+\tau \int_{1}^{e} v_{n}(x) \frac{d x}{x} \\
& +\frac{1}{\Gamma(w)} \int_{1}^{\zeta}\left(\ln \frac{\zeta}{x}\right)^{w-1} v_{n}(x) \frac{d x}{x}, \forall \ell \in[1, e] .
\end{aligned}
$$

It is enough to check that a function $v^{*} \in(\mathfrak{S E E} \mathfrak{L})_{\mathfrak{Q}, s^{*}}$ exists such that

$$
\begin{aligned}
\psi^{*}(\ell) & =-\frac{1}{\Gamma(w)} \int_{1}^{\ell}\left(\ln \frac{\ell}{x}\right)^{w-1} v^{*}(x) \frac{d x}{x}+\tau \int_{1}^{e} v^{*}(x) \frac{d x}{x} \\
& +\frac{1}{\Gamma(w)} \int_{1}^{\zeta}\left(\ln \frac{\zeta}{x}\right)^{w-1} v^{*}(x) \frac{d x}{x}, \forall \ell \in[1, e] .
\end{aligned}
$$

Assume that the continuous linear operator,

$$
\mathrm{Y}: \mathcal{L}^{1}([1, e], \Re) \rightarrow \mathcal{N}=C([1, e], \Re)
$$

is defined by

$$
\begin{aligned}
Y(v)(\ell)=s(\ell)= & -\frac{1}{\Gamma(w)} \int_{1}^{\ell}\left(\ln \frac{\ell}{x}\right)^{w-1} v(x) \frac{d x}{x}+\tau \int_{1}^{e} v(x) \frac{d x}{x} \\
& +\frac{1}{\Gamma(w)} \int_{1}^{\zeta}\left(\ln \frac{\zeta}{x}\right)^{w-1} v(x) \frac{d x}{x}, \quad \forall \ell \in[1, e]
\end{aligned}
$$

so that

$$
\begin{aligned}
\left\|\psi_{n}(\ell)-\psi^{*}(\ell)\right\| & =\|-\frac{1}{\Gamma(w)} \int_{1}^{\ell}\left(\ln \frac{\ell}{x}\right)^{w-1}\left(v_{n}(x)-v^{*}(x)\right) \frac{d x}{x}+\tau \int_{1}^{e}\left(v_{n}(x)-v^{*}(x)\right) \frac{d x}{x} \\
& +\frac{1}{\Gamma(w)} \int_{1}^{\zeta}\left(\ln \frac{\zeta}{x}\right)^{w-1}\left(v_{n}(x)-v^{*}(x)\right) \frac{d x}{x} \| \rightarrow 0
\end{aligned}
$$

as $n \rightarrow \infty$. Thus, by utilizing Theorem 2, we go to the conclusion that the operator $\mathrm{Y} \circ$ $(\mathfrak{S E L})_{\mathscr{Q}, s_{n}}$ has a closed graph. As we know that $\psi_{n} \in\left((\mathfrak{S E E})_{\mathscr{W}, s_{n}}\right)$ and $s_{n} \rightarrow s^{*}$, there exists $v^{*} \in(\mathfrak{S} \mathfrak{E} \mathfrak{L})_{\omega, s^{*}}$ such that

$$
\begin{aligned}
\psi^{*}(\ell) & =-\frac{1}{\Gamma(w)} \int_{1}^{\ell}\left(\ln \frac{\ell}{x}\right)^{w-1} v^{*}(x) \frac{d x}{x}+\tau \int_{1}^{e} v^{*}(x) \frac{d x}{x} \\
& +\frac{1}{\Gamma(w)} \int_{1}^{\zeta}\left(\ln \frac{\zeta}{x}\right)^{w-1} v^{*}(x) \frac{d x}{x}, \forall \ell \in[1, e] .
\end{aligned}
$$

Thus, $\psi^{*} \in \mathcal{A}_{2} s^{*}$ and so $\mathcal{A}_{2}$ has a closed graph. Thus, $\mathcal{A}_{2}$ is upper semi-continuous. Moreover, by using the hypothesis, the operator $\mathcal{A}_{2}$ has compact values. This implies that 
$\mathcal{A}_{2}$ is an upper semi-continuous and compact operator. Now, by using condition $\left(\mathcal{S}_{6}\right)$, we obtain

$$
\begin{aligned}
M_{0}^{*} & =\left\|\mathcal{A}_{2}(\mathcal{N})\right\|=\sup \left\{\left|\mathcal{A}_{2} s\right|: s \in \mathcal{N}\right\} \\
& =\left[\frac{1}{\Gamma(w+1)}+\tau+\frac{(\ln \zeta)^{w}}{\Gamma(w+1)}\right]\|p\|_{\mathcal{L}^{1}} \\
& =\triangle^{*}\|p\|_{\mathcal{L}^{1}} .
\end{aligned}
$$

Setting $\ell^{*}=k^{*}$, we get $M_{0}^{*} \ell^{*}<\frac{1}{2}$, and thus the assumptions of Theorem 3 are satisfied for $\mathcal{A}_{1}$ and $\mathcal{A}_{2}$. Thus, one of the conditions (i) or (ii) holds. We prove that the condition (ii) is not possible. By using Theorem 3 and the assumption $\left(\mathcal{S}_{7}\right)$, consider that $s \in O$ with $\|s\|=\tilde{\epsilon}$. Then, $v s(\ell) \in \mathcal{A}_{1} s(\ell) \mathcal{A}_{2} s(\ell)$ for each $v>1$. Choose a related function $v \in(\mathfrak{S E L})_{\mathfrak{Q}, S}$. Then, $\forall v>1$, we get

$$
\begin{aligned}
s(\ell) & =\frac{1}{v} g(\ell, s(\ell))\left[-\frac{1}{\Gamma(w)} \int_{1}^{\ell}\left(\ln \frac{\ell}{x}\right)^{w-1} v(x) \frac{d x}{x}+\tau \int_{1}^{e} v(x) \frac{d x}{x}\right. \\
& \left.+\frac{1}{\Gamma(w)} \int_{1}^{\zeta}\left(\ln \frac{\zeta}{x}\right)^{w-1} v(x) \frac{d x}{x}\right], \forall \ell \in[1, e] .
\end{aligned}
$$

Thus, we obtain

$$
\begin{aligned}
|s(\ell)| & =\frac{1}{v} g(\ell, s(\ell))\left[-\frac{1}{\Gamma(w)} \int_{1}^{\ell}\left(\ln \frac{\ell}{x}\right)^{w-1}|v(x)| \frac{d x}{x}+\tau \int_{1}^{e}|v(x)| \frac{d x}{x}\right. \\
& \left.+\frac{1}{\Gamma(w)} \int_{1}^{\zeta}\left(\ln \frac{\zeta}{x}\right)^{w-1}|v(x)| \frac{d x}{x}\right] \\
& \leq[|g(\ell, s(\ell))-g(\ell, 0)|+|g(\ell, 0)|]\left[\frac{1}{\Gamma(w)} \int_{1}^{\ell}\left(\ln \frac{\ell}{x}\right)^{w-1}|v(x)| \frac{d x}{x}+\tau \int_{1}^{e}|v(x)| \frac{d x}{x}\right. \\
& \left.+\frac{1}{\Gamma(w)} \int_{1}^{\zeta}\left(\ln \frac{\zeta}{x}\right)^{w-1}|v(x)| \frac{d x}{x}\right] \\
& \leq\left(k^{*}\|s\|+G^{*}\right)\left[\frac{1}{\Gamma(w)} \int_{1}^{\ell}\left(\ln \frac{\ell}{x}\right)^{w-1} p(x) \frac{d x}{x}+\tau \int_{1}^{e} p(x) \frac{d x}{x}\right. \\
& \left.+\frac{1}{\Gamma(w)} \int_{1}^{\zeta}\left(\ln \frac{\zeta}{x}\right)^{w-1} p(x) \frac{d x}{x}\right] \\
& \leq\left(k^{*} \tilde{\epsilon}+G^{*}\right) \triangle^{*}\|p\|_{\mathcal{L}^{1}}, \forall \ell \in[1, e] .
\end{aligned}
$$

So

$$
\tilde{\epsilon} \leq \frac{G^{*} \triangle^{*}\|p\|_{\mathcal{L}^{1}}}{1-k^{*} \triangle^{*}\|p\|_{\mathcal{L}^{1}}} .
$$

According to condition (11), we can see that the condition (ii) of Theorem 3 is not possible. Thus, $s \in \mathcal{A}_{1} s \mathcal{A}_{2} s$. Hence, it is satisfied that $\omega$ has a fixed point and so that the hybrid CH-FIBVP (3) and (4) has a solution.

We conclude from the above result that the solution for the hybrid Caputo-Hadamard fractional differential inclusion exists provided that the stated conditions hold. 


\section{Examples}

Now, we provide numerical examples to demonstrate our theoretical findings.

Example 1. Consider hybrid CH-FBVP (1) and (2). Choose $w=1.5, w-1=0.5, \zeta=1.8$ and $\tau=0.1$. Assume that the continuous maps $g:[1, e] \times \Re \rightarrow \Re /\{0\}$ and $\eta:[1, e] \times \Re \rightarrow \Re$ are defined by

$$
g(\ell, s(\ell))=\frac{\ell|s(\ell)|^{2}}{6+|s(\ell)|}+5 \text { with } G^{*}=\sup _{\ell \in[1, \ell]}|g(\ell, 0)|=5
$$

and

$$
\eta(\ell, s(\ell))=\frac{\ell \sin ^{2}\left(\frac{\pi \ell}{2}\right) \sin (s(\ell))}{1000} .
$$

Now, put $k(\ell)=\ell$ and $h(\ell)=\frac{\ell \sin ^{2}\left(\frac{\pi}{2}(\ell)\right)}{1000}$. Then, hybrid CH-FBVP takes the form

$$
C H_{\mathfrak{D}_{1}} 1^{+.5}\left(\frac{s(\ell)}{\frac{\ell|s(\ell)|^{2}}{6+|s(\ell)|}+5}\right)+\frac{\ell \sin ^{2}\left(\frac{\pi}{2}(\ell)\right) \sin (s(\ell))}{1000}=0,(\ell \in[1, e])
$$

supplemented with the three-point hybrid terminal conditions

$$
\begin{aligned}
& \left.\mathfrak{D}\left(\frac{s(\ell)}{\frac{\ell|s(\ell)|^{2}}{6+|s(\ell)|}+5}\right)\right|_{\ell=1}=0 \\
& \left.0.1^{C H} \mathfrak{D}_{1^{+}}^{0.5}\left(\frac{s(\ell)}{\frac{\ell|s(\ell)|^{2}}{6+|s(\ell)|}+5}\right)\right|_{\ell=e}+\left.\left(\frac{s(\ell)}{\frac{\ell|s(\ell)|^{2}}{6+|s(\ell)|}+5}\right)\right|_{\ell=1.8}=0 .
\end{aligned}
$$

Then, we get $k^{*}=\sup _{\ell \in[1, e]}|k(\ell)| \simeq 2.7, H^{*}=\sup _{\ell \in[1, e]}|h(\ell)|=\frac{e \sin ^{2}\left(\frac{\pi}{2}(e)\right)}{1000} \simeq$ $0.0021, \mathcal{M}(\|s\|)=1$ and $\triangle^{*} \simeq 1$ 1915. Then, $k^{*} \triangle^{*} H^{*} \mathcal{M}(\|s\|) \simeq 0.0068<1$. Choose $\varsigma>0.0126$. The hybrid CH-FBVP (13) and (14) has a solution according to Theorem 4. The graphical illustration of the inequality (9) in assumption $\mathcal{S}_{2}$ of Theorem 4 is given in Figure 1. Figures 2 and 3 depict the graphs of the functions $\eta$ and $g$, respectively.

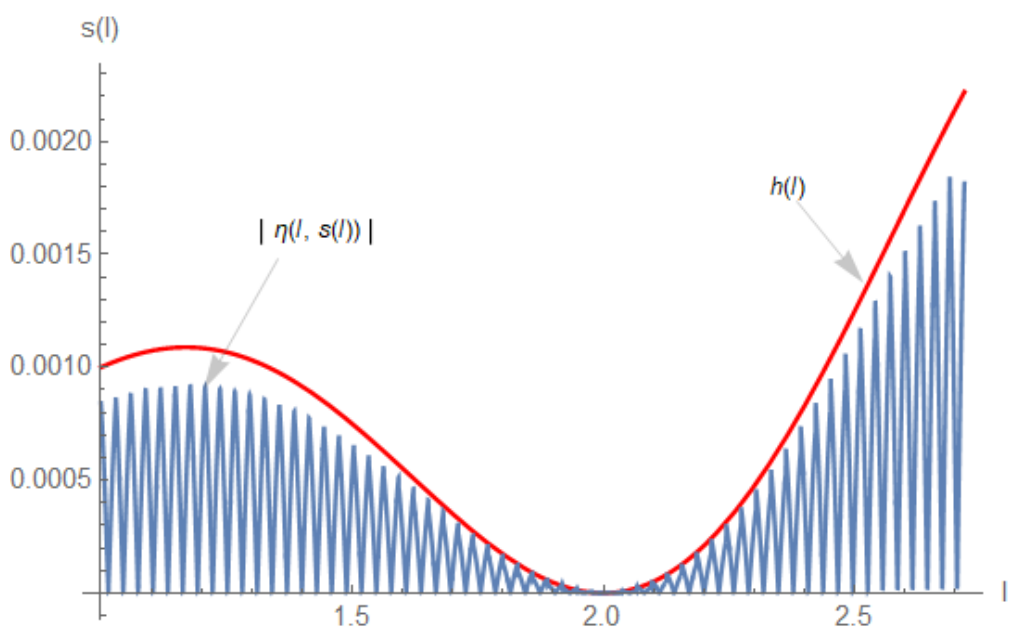

Figure 1. Graphical illustration of inequality (9). 


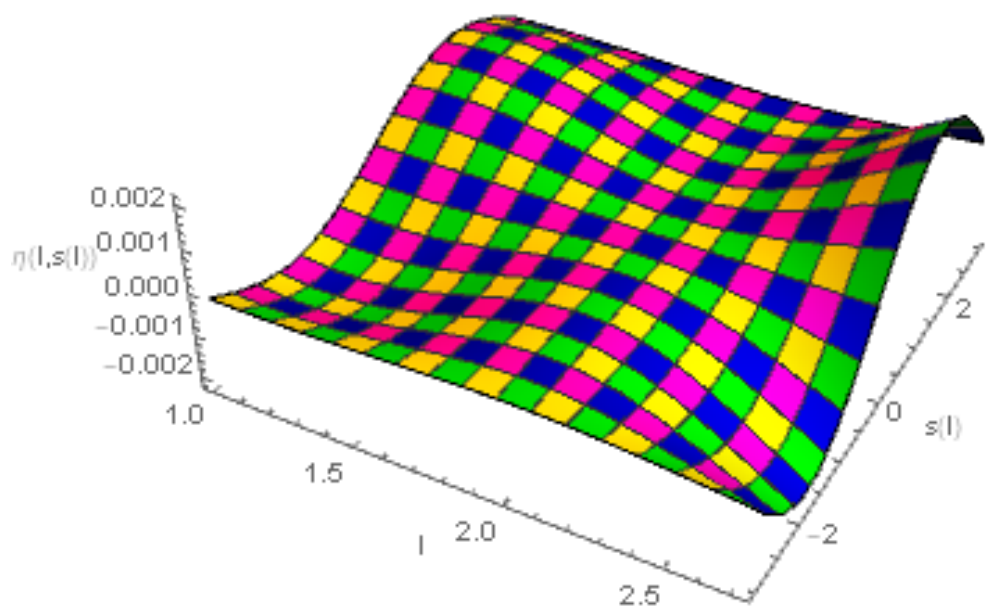

Figure 2. The graph of $\eta(l, s(l))$.

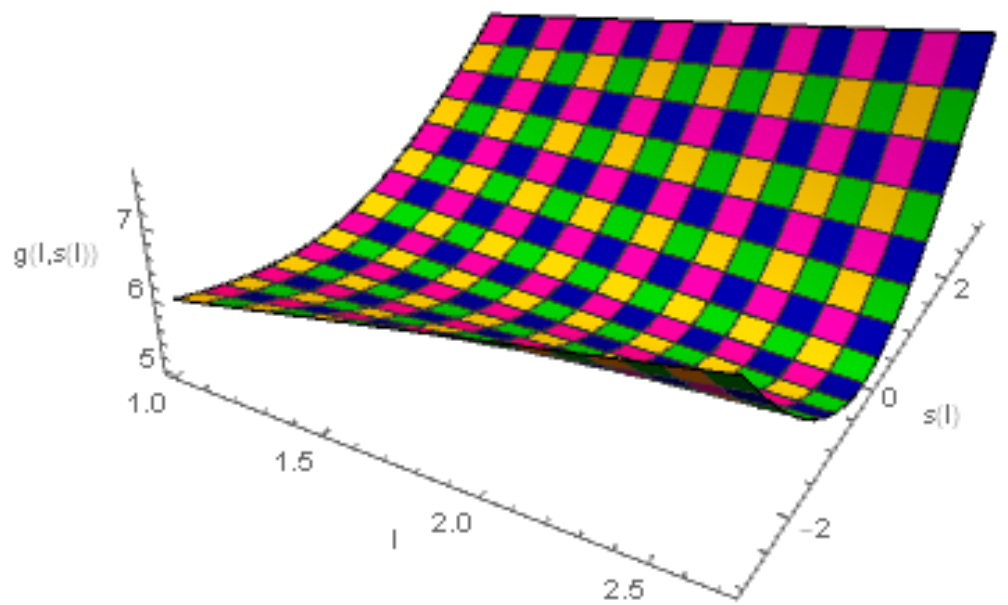

Figure 3. The graph of $g(l, s(l))$.

Example 2. Let $w=1.6, w-1=0.6, \zeta=1.89, \tau=1.75$ and a continuous map $g:[1, e] \times$ $\Re \backslash\{0\}$ defined by $g(\ell, s(\ell))=\ell \sin \frac{s(\ell)}{120}+0.008$ with $G^{*}=\sup _{\ell \in[1, e]}|g(\ell, 0)|=0.008$. Define a multi-valued map $\omega:[1, e] \times \Re \rightarrow \mathcal{I}(\Re)$ by

$$
\omega(\ell, s(\ell))=\left[\frac{|s(\ell)|}{4(|s(\ell)|+1)}+0.5, \frac{|\cos s(\ell)|^{4}}{5\left(1+|\cos s(\ell)|^{4}\right)}+1.5\right]
$$

in the proposed hybrid CH-FBVP (3) and (4), then the hybrid CH-FIBVP takes the form,

$$
C H_{\mathfrak{D}_{1+}^{1.6}}\left(\frac{s(\ell)}{\ell \sin \frac{s(\ell)}{120}+0.008}\right) \in\left[\frac{|s(\ell)|}{4(|s(\ell)|+1)}+0.5, \frac{|\cos s(\ell)|^{4}}{5\left(1+|\cos s(\ell)|^{4}\right)}+1.5\right], \quad(\ell \in[1, e])
$$

supplemented with three-point hybrid terminal conditions,

$$
\begin{aligned}
& \left.\mathfrak{D}\left(\frac{s(\ell)}{\ell \sin \frac{s(\ell)}{120}+0.008}\right)\right|_{\ell=1}=0 \\
& \left.(1.75)^{C H} \mathfrak{D}_{1^{+}}^{0.6}\left(\frac{s(\ell)}{\ell \sin \frac{s(\ell)}{120}+0.008}\right)\right|_{\ell=e}+\left.\left(\frac{s(\ell)}{\ell \sin \frac{s(\ell)}{120}+0.008}\right)\right|_{\ell=1.89}=0 .
\end{aligned}
$$




$$
\begin{aligned}
& \text { If } k(\ell)=\frac{\ell}{120}, \text { then } k^{*}=\sup _{\ell \in[1, e]}|k(\ell)|=\frac{e}{120} \simeq 0.0225 \text {. Since } \\
& \qquad|\psi| \leq \max \left(\frac{|s(\ell)|}{4(|s(\ell)|+1)}+0.5, \frac{|\cos s(\ell)|^{4}}{5\left(1+|\cos s(\ell)|^{4}\right)}+1.5\right) \leq 2
\end{aligned}
$$

$\forall \psi \in \omega(\ell, s(\ell))$. We obtain $\|\omega(\ell, s(\ell))\|=\sup \{|v|: v \in \omega(\ell, s(\ell))\} \leq 2$. Put $p(\ell)=2$ for any $(\ell \in[1, e])$. Then, $\|p\|_{\mathcal{L}^{1}}=\int_{1}^{e}|p(s)| d s=2(e-1) \simeq 3.42$. Hence we get $\triangle^{*} \simeq 2.7891$. Then $k^{*} \triangle^{*}\|p\|_{\mathcal{L}^{1}} \simeq 0.2146<\frac{1}{2}$. So we have $\tilde{\epsilon}>0.0971$. Hence, hybrid CH-FIBVP (15) and (16) has at least one solution according to Theorem 5.

\section{Concluding Remarks}

Fractional differential equations and inclusions can be used to model the many realworld problems. In this paper, we look at two new hybrid Caputo-Hadamard FBVP classes with three-point hybrid Caputo-Hadamard terminal conditions. Using Dhage's fixed point theorems, we investigated the essential requirements for the existence and uniqueness of solutions to both problems. Our results are natural extensions of the hybrid Caputo fractional model of a thermostat along with hybrid boundary conditions due to Baleanu et al. [4]. Furthermore, we provided numerical examples to support the validity of our findings. The Caputo-Hadamard fractional derivative can be used to prove the existence of solutions for more sophisticated FDEs and FDIs utilizing the fixed point theory and functional analysis.

Author Contributions: Conceptualization, M.Y. and A.H.; methodology, S.M. and A.H.; validation, R.G., A.H.; formal analysis, M.Y., R.G. and A.H.; investigation, A.H.; writing-original draft preparation, S.M.; writing-review and editing, M.Y., R.G. and A.H.; supervision, R.G. and A.H.; project administration, R.G.; funding acquisition, R.G. All authors have read and agreed to the published version of the manuscript.

Funding: This research received no external funding.

Institutional Review Board Statement: Not applicable.

Informed Consent Statement: Not applicable.

Data Availability Statement: No data were used.

Conflicts of Interest: The authors declare no conflict of interest.

\section{Abbreviations}

The following abbreviations are used in this manuscript:

$\begin{array}{ll}\mathrm{CH}_{\mathfrak{D}} & \text { Caputo-Hadamard Fractional Derivative } \\ \mathrm{CH}_{\mathfrak{I}} & \text { Caputo-Hadamard Fractional Integral } \\ \mathrm{CH}-\text { FBVP } & \text { Caputo-Hadamard Fractional Boundary Value Problem } \\ \mathrm{CH}-\text { FIBVP } & \text { Caputo-Hadamard Fractional Inclusion Boundary Value Problem } \\ \mathcal{N} & \text { Normed Space } \\ \mathcal{I}(\mathcal{N}) & \text { Set of all Subsets of } \mathcal{N} \\ P H_{d} & \text { Pompeiu-Hausdorff metric } \\ (\mathfrak{S E E} \mathfrak{L})_{\omega, \sigma} & \text { Selections of } \omega \text { at } \sigma\end{array}$

\section{References}

1. Hilfer, R. Application of Fractional Calculus in Physics; World Scientific: Singapore, 2000.

2. Kilbas, A.A.; Srivastava, H.M.; Rujillo, J.J. Theory and Applications of Fractional Diferential Equations; Elsevier: Amsterdam, The Netherlands, 2006.

3. Mohammadi, H.; Rezapour, S. Two existence results for nonlinear fractional diferential equations by using fixed point theory on ordered gauge spaces. J. Adv. Math. Stud. 2013, 6, 154-158. 
4. Baleanu, D.; Etemad, S.; Rezapour, S. A hybrid Caputo fractional modeling for thermostat with hybrid boundary value conditions. Bound. Value Probl. 2020, 2020, 64. [CrossRef]

5. Baleanu, D.; Nazemi, S.Z.; Rezapour, S. Attractivity for a k-dimensional system of fractional functional diferential equations and global attractivity for a k-dimensional system of nonlinear fractional diferential equations. J. Inequal. Appl. 2014, $2014,31$. [CrossRef]

6. Baleanu, D.; Nazemi, S.Z.; Rezapour, S. The existence of solution for a k-dimensional system of multi-term fractional integrodiferential equations with anti-periodic boundary value problems. Abstr. Appl. Anal. 2014, 2014, 896871. [CrossRef]

7. Wang, G.; Ren, X.; Zhang, L.; Ahmad, B. Explicit iteration and unique positive solution for a Caputo-Caputo-Hadamard fractional turbulent fow model. IEEE Access 2019, 7, 109833-109839. [CrossRef]

8. Wang, G.; Pei, K.; Chen, Y.Q. Stability analysis of nonlinear Caputo-Hadamard fractional diferential system. J. Franklin Inst. 2019, 356, 6538-6546. [CrossRef]

9. Wang, G.; Pei, K.; Agarwal, R.P.; Zhang, L.; Ahmad, B. Nonlocal Caputo-Hadamard fractional boundary value problem with Caputo-Hadamard integral and discrete boundary conditions on a half-line. J. Comput. Appl. Math. 2018, 343, 230-239. [CrossRef]

10. Pei, K.; Wang, G.; Sun, Y. Successive iterations and positive extremal solutions for a Caputo-Hadamard type fractional integrodiferential equations on infnite domain. Appl. Math. Comput. 2017, 312, 158-168.

11. Abbas, S.; Benchohra, M.; Hamani, S.; Henderson, J. Upper and lower solutions method for Caputo-Hadamard fractional differential inclusions. Math. Moravica 2019, 23, 107-118. [CrossRef]

12. Benchohra, M.; Graef, J.R.; Guerraiche, N.; Hamani, S. Nonlinear boundary value problems for fractional differential inclusions with Caputo-Hadamard derivatives on the half line. AIMS Math. 2016, 6, 6278-6292. [CrossRef]

13. Hamani, S.; Henderson, J. Boundary value problems for fractional differential inclusions with nonlocal conditions. Mediterr. J. Math. 2016, 13, 967-979. [CrossRef]

14. Benhamidaa, W.; Hamania, S.; Hendersonb, J. Boundary Value Problems For Caputo-Hadamard fractional differential equations. Adv. Theory Nonlinear Anal. Its Appl. 2018, 2, 138-145.

15. Benchohra, M.; Hamani, S.; Zhou, Y. Oscillation and nonoscillation for Caputo-Hadamard impulsive fractional differential inclusions. Adv. Differ. Equations 2019, 2019, 74. [CrossRef]

16. Dhage, B.C.; Lakshmikantham, V. Basic results on hybrid diferential equation. Nonlinear Anal. Hybrid Syst. 2010, 4, 414-424 [CrossRef]

17. Zhao, Y.; Sun, S.; Han, Z.; Li, Q. Theory of fractional hybrid diferential equations. Comput. Math. Appl. 2011, 62, 1312-1324. [CrossRef]

18. Sun, S.; Zhao, Y.; Han, Z.; Li, Y. The existence of solutions for boundary value problem of fractional hybrid differential equations. Commun. Nonlinear Sci. Numer. Simul. 2012, 17, 4961-4967. [CrossRef]

19. Hilal, K.; Kajouni, A. Boundary value problems for hybrid diferential equations with fractional order. Adv. Differ. Equations 2015, 2015, 183. [CrossRef]

20. Baleanu, D.; Hedayati, V.; Rezapour, S.; Al Qurashi, M.M. On two fractional diferential inclusions. SpringerPlus 2016,5 , 882. [CrossRef] [PubMed]

21. Infante, G.; Webb, J. Loss of positivity in a nonlinear scalar heat equation. Nonlinear Differ. Equ. Appl. 2006, 13, 249-261. [CrossRef]

22. Nieto, J.J.; Pimentel, J. Positive solutions of a fractional thermostat model. Bound. Value Probl. 2013, 2013, 5. [CrossRef]

23. Miller, K.S.; Ross, B. An Introduction to Fractional Calculus and Fractional Diferential Equations; Wiley: New York, NY, USA, 1993.

24. Deimling, K. Multi-Valued Diferential Equations; de Gruyter: Berlin, Germany, 1992.

25. Aubin, J.; Cellina, A. Diferential Inclusions: Set-Valued Maps and Viability Theory; Springer: Berlin, Germany, 1984.

26. Dhage, B.C. Nonlinear functional boundary value problems involving Carathedory. Kyungpook Math. J. $2006,46,427-441$.

27. Lasota, A.; Opial, Z. An application of the Kakutani-Ky Fan theorem in the theory of ordinary diferential equations. Bull. Acad. Pol. Sci. Set. Sci. Math. Astronom. Phys. 1965, 13, 781-786.

28. Dhage, B.C. Existence results for neutral functional diferential inclusions in Banach algebras. Nonlinear Anal. 2006, 64, 1290-1306. [CrossRef] 